\title{
Management of common gastrointestinal disorders: quality criteria based on patients' views and practice guidelines
}

\author{
Roger Jones, Claire Hunt, Richard Stevens, Jamie Dalrymple, Richard Driscoll,
} Sarah Sleet and Jonathan Blanchard Smith

\section{ABSTRACT}

\section{Background}

Although gastrointestinal disorders are common in general practice, clinical guidelines are not always implemented, and few patient-generated quality criteria are available to guide management.

Aim

To develop quality criteria for the management of four common gastrointestinal disorders: coeliac disease, gastro-oesophageal reflux disease (GORD), inflammatory bowel disease, and irritable bowel syndrome.

\section{Design of study}

Qualitative study including thematic analysis of transcripts from patient focus groups and content analysis of published clinical practice guidelines. Emergent themes were synthesised by a consensus panel, into quality criteria for each condition.

Setting

Community-based practice in England, UK.

Methods

Fourteen focus groups were conducted (four for coeliac disease, irritable bowel syndrome, and inflammatory bowel disease, and two for GORD) involving a total of 93 patients (64 females, 29 males; mean age 55.4 years). Quality criteria were based on patients' views and expectations, synthesised with an analysis of clinical practice guidelines.

\section{Results}

A chronic disease management model was developed for each condition. Key themes included improving the timeliness and accuracy of diagnosis, appropriate use of investigations, better provision of information for patients, including access to patient organisations, better communication with, and access to, secondary care providers, and structured follow-up and regular review, particularly for coeliac disease and inflammatory bowel disease.

\section{Conclusion}

This study provides a model for the development of quality markers for chronic disease management in gastroenterology, which is likely to be applicable to other chronic conditions.

\section{Keywords}

gastrointestinal diseases; primary healthcare; quality of healthcare.

\section{INTRODUCTION}

Variation in the quality of care provided by individual clinicians, clinical teams, and institutions has been a feature of the medical landscape for decades. Attempts to identify unevenness in the provision and outcomes of care began with medical audit, introduced by Donabedian in the 1970 s, ${ }^{1}$ with an explosion of interest in evidence-based medicine, ${ }^{2,3}$ and a proliferation of evidence-based guidelines. However, the problems of getting evidence into practice, or guidelines into action, have been widely documented, ${ }^{4,5}$ so that a new discipline of 'implementation research' has emerged to deal with the problem. . $^{6}$

In a bold experiment, the NHS in the UK cut many of the implementation corners when it introduced, as part of GPs' contract, the Quality and Outcomes Framework (QOF) in 2004. ${ }^{\circ}$ GPs score QOF points for attaining targets related to the management of common chronic disorders, and their score is

R Jones, DM, FRCGP, Wolfson professor of general practice; C Hunt, BSc(Hons), MSc, research fellow, Department of General Practice and Primary Care, King's College London. R Stevens, FRCGP, GP, Oxford. J Dalrymple, FRCGP, chairman, Primary Care Society for Gastroenterology, Norwich. R Driscoll, M.Litt, MSc, chief executive, National Association for Colitis and Crohn's Disease, St Albans. S Sleet, BA, BSc(Hons), chief executive, Coeliac UK, High Wycombe. J Blanchard Smith, BA(Hons), M.Phil, Chair, The Gut Trust, Sheffield.

Address for correspondence

Professor Roger Jones, Department of General Practice and Primary Care, 5 Lambeth Walk, London SE11 6SP.

E-mail: roger.jones@kcl.ac.uk

Submitted: 18 August 2008; Editor's response: 8 October 2008; final acceptance: 4 November 2008.

()British Journal of General Practice.

This is a full-length article of an abridged version published in print and originally published online first on $28 \mathrm{Apr} 2009$. Cite this version as: Br J Gen Pract 2009 DOI: 10.3399/bjgp09X420761 (abridged text, in print: Br J Gen Pract 2009; 59: 415-421). 
translated directly into a significant proportion of their income. Although the government underestimated the quality of care before introducing the $\mathrm{QOF},{ }^{10}$ so that the scheme was more expensive than anticipated, it seems that pay-for-performance, at least in the UK, is here to stay, not least because evidence is accumulating to show that standards of care in some QOF domains are rising more rapidly than they were before the QOF was introduced. ${ }^{11-14}$

Although the QOF is regularly updated and expanded, it does not cover all important clinical problems and has not yet included any recognition of chronic gastrointestinal disorders managed in primary care. The QOF has been criticised, with some justification, for focusing on the 'what' rather than the 'how' of quality improvement, and on readily measured metrics that relate to processes of care and to proxy outcomes. Despite attempts to ensure user involvement in defining QOF criteria, it has been argued that this approach does not recognise much of the psychosocial content of general practice care and may deflect GPs' attention from important aspects of patient management that are not directly remunerated. ${ }^{15,16}$

Patient engagement and choice, and the importance of the 'user voice' in planning and evaluating health care have achieved prominence in the NHS. Medical researchers are also being encouraged to consider more carefully how they might measure the benefits to patients of their work, both through the UK National Institute of Health Research's Research for Patient Benefit Programme, ${ }^{17}$ and also through the Food and Drugs Administration in the US, where the inclusion of patient-reported outcomes in the design and reporting of clinical trials of new therapies is now mandatory. ${ }^{18}$

The present study has been carried out to address the need for more patient-centred quality criteria, particularly for chronic gastrointestinal disorders, in which patients' experiences and views about the diagnosis and management of four common gastrointestinal disorders - gastro-oesophageal reflux disease (GORD), irritable bowel syndrome, coeliac disease, and inflammatory bowel disease have been collected. These experiences and views have been synthesised with digests of clinical evidence and clinical practice guidelines on the management of these disorders, to generate a set of quality criteria for each of them. This work forms the basis of the IMAGE - Improving Management in Gastroenterology - project, one of a suite of nine quality-improvement projects funded by the Health Foundation in the UK, in which evidence-based computer decision support will be evaluated in general practices as a means of improving the quality of care of common gastrointestinal disorders.

\section{How this fits in}

Gastrointestinal disorders account for about $10 \%$ of the clinical work of the NHS and there is evidence that they are not always well managed in general practice. Few quality criteria are available to guide their management. This study has synthesised the views of patients about diagnosis and management, with evidence-based recommendations distilled from clinical practice guidelines to produce a set of quality criteria for the management of irritable bowel syndrome, gastro-oesophageal reflux disease, coeliac disease, and inflammatory bowel disease. These criteria provide guidance on clinical management and also represent the results of a methodology for identifying quality criteria, which may be valuable in other long-term conditions.

\section{METHOD}

The study was conducted in south east London, Essex, Bristol, Oxford, Norfolk, Teeside, and Sunderland, UK and was carried out in three stages:

- patient focus groups;

- literature review and guideline synthesis; and

- quality criteria setting process

The study was carried out by a multidisciplinary team. An implementation group was formed consisting of a core multi-disciplinary team at King's College London, plus a number of primary care physicians and the chief executives of three national patient organisations: the National Association for Colitis and Crohn's Disease; the Gut Trust (for irritable bowel syndrome); and Coeliac UK, the national organisation for coeliac disease. This group met frequently to plan and manage the study, which was overseen by a larger advisory board consisting of the group plus a professor of gastrointestinal nursing, a health economist, two specialist gastroenterologists, and the chief executive of CORE (the working name of the Digestive Disorders Foundation). 'GastroChampions' were appointed as principal investigators at each study site, whose responsibility was to secure and maintain the involvement of local general practices. These were experienced GPs with established expertise in primary care gastroenterology.

\section{Patient focus groups}

Patients who were known to have the index conditions were invited to participate in focus groups conducted by an experienced facilitator, either by invitation from their own GP (the local principal investigator at each study site) or from the relevant patient organisation. Four focus groups, two in each study site, were conducted for the three conditions with a national patient organisation, and two focus groups were conducted for GORD, one at each study 


\section{Box 1. Key points emerging from the inflammatory bowel disease focus groups.}

\section{Main points}

- Information for patients:

\section{- GP/information sheets}

- Information about consultants

Education for GPs and hospital doctors

- Access to GP, hospital (hotline), and GP with special interest in gastroenterology

- Inflammatory bowel disease nurse, practice nurse, stoma nurse

- Dietician

- National Association for Colitis and Crohn's Disease support groups

- Structured, shared and coordinated care: confidence in coordination:

\section{- Shared care record system}

\section{- Coordination of blood tests}

\section{Additional discussion points}

- Generic drugs and branded drugs

- Alternative/complementary and alternative medicine treatments: probiotics, aloe vera, acupuncture, reiki

- Radar key (access to public lavatories)

Disability badges, insurance

site so that a total of 14 focus groups were conducted between October 2007 and January 2008.

The focus groups used a topic guide developed on the basis of discussions between clinical colleagues working on the project and the patient organisations. The groups were all run by the same experienced facilitator, lasted 1-2 hours, and were audiotaped,

\section{Box 2. Key themes on diagnosis and management from inflammatory bowel disease guidelines.}

- Early and accurate diagnosis, distinction from irritable bowel syndrome, investigation of patients with persistent symptoms, especially pain, diarrhoea, rectal bleeding, mucus

- Practice database for structured follow-up and audit

- Patient information Patient UK, National Association for Colitis and Crohn's Disease (especially their audit), CORE

- Clear explanation, support, prognosis, access to primary and secondary care advice

- Clear arrangements for follow-up - physician and psychosocial impact

- Access to/contact with specialist services: inflammatory bowel disease consultant/nurse/dietician/pregnancy and contraception

- Dietary and lifestyle (especially smoking) advice

- Shared care of inflammatory bowel disease - shared record especially regarding immunosuppressant treatment (full blood count) and steroids (bone mineral density), surgery, postoperative care transcribed, and analysed manually using qualitative methodology. Thematic analysis was used to identify emerging themes, which were redefined iteratively, and triangulated between at least two of the study investigators, and were also read by the focus group facilitator to ensure accuracy.

\section{Literature review and guideline synthesis}

A review was carried out (using electronic searches of standard medical databases, supplemented by searching the internet) of current guidelines on the management of the four index conditions in which English language guidelines were identified that had been developed by gastroenterological bodies (such as the Primary Care Society for Gastroenterology, the British Society of Gastroenterology, the American Gastroenterological Association, and the World Gastroenterology Organisation); guideline development groups (such as the National Institute for Health and Clinical Excellence [NICE] and the Scottish Intercollegiate Guidelines Network [SIGN]); patient organisations (such as CORE, the digestive diseases charity); and patient information sites (such as Patient UK). Recommendations relevant to diagnosis and management in primary care were extracted from all these documents and summarised for circulation to the advisory board and GastroChampions for discussion at a day-long consensus meeting as described below.

\section{Quality criteria-setting process}

The major themes emerging from the focus groups were summarised, along with the main points relating to diagnosis and management extracted from the literature and guidelines reviews. A provisional list of key points derived from both the patient groups and the guideline review, numbering between 10 and 20 for each condition, was assembled, supplemented by secondary points for further discussion and clarification. This information was circulated to the advisory board and GastroChampions, who convened for a 1-day meeting where 2 hours were devoted to each condition, during which this information was distilled into a final list of eight to ten quality criteria for each of the four index conditions. A modified Delphi approach was then used, in which these lists were circulated twice more for confirmation and further email discussion to derive an agreed final list. These will then be presented to participating general practices in which they will be implemented in a further phase of the project, using a multi-faceted intervention based on the use of computer prompts and templates.

\section{RESULTS}

Results are presented in three sections, representing the three stages of the project. The focus group 
results (Box 1) and guideline analysis (Box 2) for one of the conditions (inflammatory bowel disease) are shown as illustrations, and the final sets of quality criteria for all four conditions are shown in Boxes 3-6). The results of the other focus group and guideline analyses for GORD, irritable bowel syndrome, and coeliac disease are shown in Appendices 1-6.

\section{Focus groups}

Fourteen focus groups were conducted, involving 93 patients (64 females and 29 males), with a mean age of 55.4 years (range 18-82 years). A number of the emerging themes were common to all four conditions; in addition a number of disease-specific themes emerged. Particularly striking was the observation that most patients with these conditions had not previously had the opportunity to discuss them with fellow patients, and many were unaware of the existence of the relevant patient organisation for their condition or the availability of authoritative patient information material. Other general themes included the need for informed, interested, and timely diagnosis and management in primary care, and for the condition to be taken seriously, and not trivialised. Clear explanation and prognosis were both seen as essential. Communication and coordination with secondary care specialists, including specialist nurses and dieticians, was also highly valued by patients. Patients clearly found these meetings beneficial and have subsequently collaborated further with the researchers in helping to develop some of the study documentation, including assisting with the validation of a questionnaire developed to obtain information on health service use and personal costs of care.

\section{Clinical practice guidelines}

The literature review involved identification, analysis, and collation of over 180 documents, and key features relating to diagnosis and management in primary care are summarised in Box 2 and Appendices 4-6. A full list of these guidelines is available. The striking overall conclusion from this review was that these four conditions all conformed to a chronic disease-management model, with coeliac disease and inflammatory bowel disease in particular meeting criteria for structured primary secondary care management, including the establishment of a practice register, annual/periodic review, records shared between primary and secondary care, and access to, and easy communication between, primary care and specialist services.

\section{Quality criteria}

Results of the consensus meeting at which the patient

\section{Box 3. Quality criteria for inflammatory bowel disease.}

- On practice register

\section{Diagnosis}

Documented diagnosis

Patient

- Patient information given

- Patient support group details provided

- Smoking advice provided

Management

- On 5-aminosalicylic acid (5-ASA)

Adherence assessed

- Symptom control assessed

Quality of life assessed

- Depression excluded

- Annual review ${ }^{\mathrm{a}}$

Access to hospital/inflammatory bowel disease nurse for flare-ups

Access to dietary advice

- Complementary and alternative therapies discussed

${ }^{a}$ Checklist of annual/periodic follow up items: full blood count, C-reactive protein (CRP), liver function tests (and renal function if on 5-ASA), endoscopic surveillance according to local policy, monitoring if on immunosuppressives, symptom control.

\section{Box 4. Quality criteria for irritable bowel syndrome.}

\section{Diagnosis}

- Positive diagnosis made

- Alarm symptoms excluded

- Full blood count/erythrocyte sedimentation rate/coeliac screen completed

- Predominant symptoms established: constipation/diarrhoea/alternating (IBSC/IBS-D/IBS-A)

\section{Patient}

- Patient information given

- Patient support group details provided

- Diet discussed and advice given

\section{Management}

- Regular review offered

- Symptom control assessed

- Depression excluded

- Quality of life assessed

- Tricyclic/selective serotonin reuptake inhibitor treatment considered

- Cognitive behavioural therapy or other psychological intervention considered/offered at 12 months. 


\section{Box 5. Quality criteria for coeliac disease.}

On practice register

\section{Diagnosis}

- Diagnosis made on the basis of serology (anti-endomysium antibodies/tissue transglutaminase antibodies)

- Diagnosis confirmed on duodenal biopsy

\section{Patient}

- Patient information given

- Patient support group details provided

Gluten-free diet/products discussed

\section{Management}

- Gluten-free prescriptions arranged

Annual follow-up carried out ${ }^{\mathrm{b}}$

Symptom control assessed

- Quality of life assessed

- Depression excluded

- Access to dietician

a Make contact/liaise with the community pharmacist if necessary to ensure prescription items are readily available. ${ }^{\text {}} \mathrm{C}$ hecklist of annual follow-up items: full blood count, thyroidstimulating hormone, calcium, liver function tests, B12, folate, ferritin, symptom assessment, osteoporosis investigation in line with local screening criteria, access to dietician if needed, diet and lifestyle advice.

\section{Box 6. Quality criteria for GORD}

\section{Diagnosis}

- Diagnosis made on cardinal clinical symptoms (heartburn and regurgitation)

- Alarm symptoms excluded

\section{Patient}

- Patient information given

- Smoking, obesity, and other lifestyle advice provided

\section{Management}

On treatment with proton pump inhibitor and/or antacid

- Symptom control assessed

- Depression excluded

- Check for extra-oesophageal symptoms, such as cough/asthma

- Consider possibility of complications

GORD = gastro-oesophageal reflux disease could usefully be presented to GPs in the form of a computer-held template was 10-12 for each condition, and the iterations of the final list involved ranking candidate criteria and agreeing on the final list of criteria for which there was most support. Each criterion was linked to computer-held information including addresses and websites for patient information, details of the content of periodic/annual reviews, and validated symptom-assessment tools and quality of life measures for GPs wishing to explore these constructs in more detail with patients.

\section{DISCUSSION}

\section{Summary of main findings}

This study has succeeded in bringing together patient-generated and evidence-based criteria for the management of four common chronic disorders in primary care, to create a series of quality indicators suitable for incorporation into general practice records systems, using computer templates. The authors believe that this methodology, and the outcomes that have been reported, represent a significant improvement on previous approaches to incorporating patients' views and expectations into evidence-based medicine, and represent a valuable model for the enhancement of current quality measures and the development of new ones. These indicators also have the potential for being used to monitor both variations between practices and the quality of care provided for the four index conditions, and also to improve standards overall through the use of targets for some of them.

\section{Strengths and limitations of the study}

The strengths of this study rest largely on the central role of patients and patient organisations and the identification, through the focus group discussions, of a series of generic and specific patient-centred factors regarded as important by patients in the management of their gastrointestinal condition. This is in line with recommendations from regulatory bodies such as the Food and Drugs Authority in the US to incorporate patient-reported outcomes in clinical trials of new therapies. ${ }^{18}$ The present analysis of published clinical practice guidelines, and distillation of the most salient recommendations for management, has ensured that full attention has been paid to current evidence. The synthesis, through an expert consensus process, of patients' views and evidence-based practice guidelines is a further innovative and strong feature of the project.

Limitations of the study include the lack of a formal scoring system to rank the importance of patients' views and the main points identified in the clinical practice guidelines, with an inevitable possibility of an element of subjectivity and uneven representation of 
the views of individual members of the expert consensus group. However, a modified Delphi approach was used to allow further refinement of the quality criteria by circulating the output of the criteriasetting meeting to participants and other members of the project advisory board for confirmation and modification. It should also be acknowledged that a number of potentially important and controversial issues did not find their way into the final list of criteria. Examples for GORD might include the role of endoscopy in the investigation of patients with reflux symptoms, the role of oesophageal manometry and pHmetry, and the relevance and management of Barrett's oesophagus. It was found to be impossible to encapsulate guidance on many other important topics into a measurable, evidence-based recommendation. In other cases it was found that the evidence was contradictory or missing altogether.

\section{Comparison with existing literature}

The focus groups were arranged in different parts of the country to ensure that patients with experiences of different models of care were represented, particularly in terms of primary-secondary care relationships, and their output reflected both common themes concerning patients' experiences of diagnosis and management, and also differences in emphasis in the relationship between long-term management in general practice and in hospital. Focus groups were particularly valuable in identifying generic issues of importance to patients, notably the need for better explanations and more written information from clinicians, access to patient organisations and patient groups, and consistency and coordination between general practice and hospital management, as well as identifying diseasespecific concerns and recommendations for defining outcomes that were relevant to patients. As Fudge and colleagues have recently reported, ${ }^{19}$ patients' experience of service use are valuable in improving service delivery, although when this involvement is 'professionally determined', its value may be compromised. In the present study the focus was on patients' experience of their illness and its management to identify key areas where diagnosis, treatment, and communication could be improved. The authors consider that the 'empty ritual' of user consultation was avoided, ${ }^{20}$ and, because the patient groups were established specially for this study, much more topic-specific input was derived than they might have obtained from a 'standing group' of generic service users. ${ }^{21}$

The indicators that have been identified may be suitable for inclusion in future versions of the QOF, which consists of an evolving collection of performance indicators. During the three changes that have been made to the QOF since its inception, the trend has been to reduce the total number of indicators (from an initial 147 in 2004/2005 to 126 in $2008 / 2009$ ), to increase the proportion of QOF points, and hence the financial rewards, for achievement of the clinical components of the QOF, and to increase the number of long-term conditions included in the clinical component (from 10 to the current 19).

The QOF at present does not include targets for any gastrointestinal conditions, and the reasons for this are unclear. The criteria for inclusion in the QOF are threefold: that the management rests principally with the primary care team, that there is good evidence that health benefits are likely to arise from improved primary care, and that the disorder in question is a national priority. ${ }^{9}$ Given the ubiquity and impact of the gastrointestinal conditions that have been studied here, their inclusion in future versions of the QOF appears to be a rational proposition, not least because of the extent to which the management of many common disorders conforms to a chronic disease-management model. Tylee and Walters have made a similar argument for depression, a condition in which symptoms, disability, and patient satisfaction have been shown to improve in trials of chronic disease management. ${ }^{22}$

\section{Implications for future research and clinical practice}

The authors consider that the methodology used in this study to bring together patients' views with evidence-based guidelines represents a significant advance on current methodologies for clinical standard setting. The development of a chronic disease-management model for the care of these disorders represents a further advance, and many of this study's quality criteria can readily be translated into metrics for the quantitative evaluation of quality of care. The authors believe that not only should these, and perhaps other, chronic gastrointestinal disorders be considered for inclusion in further iterations of the QOF, but also that the present study represents a valuable, new approach to enhancing the quality of clinical management of chronic disorders, by providing guidance to clinicians on the means of achieving patient-centred and clinically appropriate outcomes, as well as a way of measuring them. Future research is required to evaluate the impact of these criteria on quality of care and to determine the utility of the methodology reported here in other conditions.

\section{Funding body}

The Health Foundation. Engaging with Quality in Primary Care programme 933/4489

\section{Ethics committee}

The study received approval from Leicestershire, Northamptonshire and Rutland Research Ethics Committee (07/H0402/56) 


\section{Competing interests}

The authors have stated that there are none

\section{Acknowledgements}

We are extremely grateful to all the patients who took part in the study, and the representatives of the patien organisations involved. We would also like to express our gratitude to all the GPs and their practice and university staff who helped us organise the focus groups, particularly our 'GastroChampions', Dr Simon Stockley, Professor Greg Rubin, Dr Michael Cohen, Dr Stevan Fox, and Professor Pali Hungin. Thank you also to John Gray of Framework UK who facilitated all the focus groups. We are very grateful to $\mathrm{Dr}$ Mark Ashworth for comments on earlier drafts of this paper, and to Pat Taylor, IMAGE project manager, for her valuable assistance. The Health Foundation provided additional support for team meetings and development.

\section{Discuss this article}

Contribute and read comments about this article on the Discussion Forum: http://www.rcgp.org.uk/bjgp-discuss

\section{REFERENCES}

1. Donabedian A. The quality of medical care. Science $1978 ; 200(4344)$ 856-864

2. G Guyatt, D Cook, B Haynes. Evidence based medicine has come long way. BMJ 2004; 329(7473): 990-991.

3. Polmear A (ed). Evidence-based diagnosis in primary care. Edinburgh: Butterworth Heinemann Elsevier, 2008.

4. Bero LA, Grilli R, Grimshaw JM, et al. Closing the gap between research and practice: an overview of systematic reviews of interventions to promote the implementation of research findings. BMJ 1998; 317(7156): 465-468.

5. Haynes B, Haines A. Barriers and bridges to evidence based clinical practice. $B M J 1998 ; 317(7153):$ 273-276.

6. Lilford RJ, Pauker SG, Braunholtz DA, Chard J. Decision analysis and the implementation of research findings. BMJ 1998; 317(7155): 405-409.

7. Grol R, Dalhuijsen, Thomas S, et al. Attributes of clinical guidelines that influence use of guidelines in general practice: observational study. BMJ 1998; 317(7162): 858-862.

8. Russell J, Greenhalgh T, Boynton P, Rigby M. Soft networks for bridging the gap between research and practice: illuminative evaluation of CHAIN. BMJ 2004; 328(7499): 1174

9. NHS: The Information Centre. Quality and Outcomes Framework. Statistics and data collections. http://www.ic.nhs.uk/statistics-and-datacollections/audits-and-performance/the-quality-and-outcomesframework (accessed 3 Mar 2009).

10. Campbell SM, Roland MO, Middleton E, Reeves D. Improvements in quality of clinical care in English general practice 1998-2003; longitudinal observational study. BMJ 2005; 331(7525): 1121-1125.

11. Steel I, Maisey S, Clark A, et al. Quality of clinical primary care and targeted incentive payments: an observational study. Br J Gen Pract 2007; 57(539): 449-454.

12. Gulliford MC, Ashworth M, Robotham D, Mohiddin A. Achievement of metabolic targets for diabetes by English primary care practices under a new system of incentives. Diabet Med 2007; 24(5): 505-511.

13. Khunti K, Gadsby R, Millett C, et al. Quality of diabetes care in the UK: comparison of published quality-of-care reports with results of the Quality and Outcomes Framework for Diabetes. Diabet Med 2007; 24(12): 1436-1441.

14. Maisey S, Steel N, Marsh R, et al. Effects of payment for performance in primary care: qualitative interview study. J Health Serv Res Policy 2008; 13(3): 133-139.

15. Lipman T. Singing from the same QOF hymn sheet: Stairway to Heaven or Mephisto Waltz? Br J Gen Pract 2006; 56: 819-820.

16. Mangin D, Toop L. The Quality and Outcomes Framework: what have you done to yourselves? Br J Gen Pract 2007; (57)539: 435-437.

17. National Institute of Health Research. The Research for Patient Benefit Programme. http://www.nihr-ccf.org.uk/site/ programmes/rfpb/default.cfm (accessed 22 Jan 2009).

18. US Food and Drug Administration. The importance of patient-reported outcomes ... it's all about the patients. http://www.fda. gov/fdac/features/2006/606_patients.html (accessed 20 Feb 2009).]

19. Fudge N, Wolfe C, McKevitt C. Assessing the promise of user involvement in health service development: ethnographic study. BMJ 2008; 336(7639): 313-317.

20. Craig GM. Involving users in developing health services. Representation is not enough: voices must be translated into action. BMJ 2008; 336(7639): 286-287.

21. Florin D, Dixon J. Public involvement in health care. BMJ 2004 328(7432): 159-161

22. Tylee $A$, Walters $P$. We need a chronic disease management model for depression in primary care. Br J Gen Pract 2007; (57)538: 348-350. 
Appendix 1. Key points emerging from the coeliac disease focus groups.

Main points

GP:

- Awareness of symptoms and groups at risk of coeliac disease

- Expertise in diagnosis

- Diagnostic distinction from irritable bowel syndrome: key/suggestive symptoms; for example diarrhoea, anaemia, abdominal symptoms

- Follow-up:

- Anaemia screening

- Structured primary/secondary care, shared care

- Dietary advice

- Bone density measurement

Patient information:

- Coeliac UK

- Patient UK information sheets

Dietary advice:

- Gluten-free products

- Costs

- Prescription

- Availability on prescription

- Eating out

- Pharmacist's role

- Labelling food

Role of specialist nurse

Additional discussion points

- Skin problems, malignancy

- Role and experience of endoscopy

- Paediatric to adult care transition

- Unnecessary and necessary endoscopies (that is, for duodenal biopsy)

Links to an eating disorder 


\section{Appendix 2. Key points emerging from the GORD focus} groups.

\section{Main points}

- Need to make an early diagnosis (demand for tests: endoscopies and biopsies)

- Chest pain suggestive of cardiac problems needs to be addressed

- Endoscopy - many patients had undergone endoscopies and seem to consider them important. Probably reflects the lack of awareness of endoscopy-negative reflux at the time

- Impact on lifestyle - needs to be taken seriously, particularly nocturnal symptoms, posture-related symptoms, and impact on social and work activities

- Reasonable support for the use of the GORD Impact Scale to achieve this

- Treatment - important to provide a range of treatments, including longterm maintenance therapies and short-term, quick-acting symptomatic treatments; for example, Gaviscon

- Importance of patient information and patient groups emphasised by some, although less strongly than for other conditions

\section{- Additional discussion points}

- Structured follow-up/shared primary-secondary care not an issue for GORD, although information from the GP and from websites and contact with other patients would be valued, but not as strongly as for the other conditions

- Some patients mentioned stress/psychological factors impacting on GORD - possibly worth considering

\section{Appendix 3. Key points emerging from the irritable bowel} syndrome focus groups.

\section{Main points}

- GPs:

- Information needed: irritable bowel syndrome as an illness, frequently accompanied by multiple, non-gastrointestinal symptoms

- Awareness of patients' fears of cancer (family history often relevant)

- Recognition of links with stress and psychiatric problems

- Need to make a positive diagnosis

- Recognition of post-infective irritable bowel syndrome

- Physical versus non-physical/stress-related causes: being taken seriously

- Information, explanation/support groups/Gut Trust ${ }^{a}$

- Dietician, self-management and links with gluten sensitivity - misconceptions

- Follow-up/review

- Role of drugs in symptom control

- Referral to expert, access to specialist

\section{Additional discussion points}

- Role of alternative treatments, for example yoga, Tai Chi, homeopathy, acupuncture

- Over-investigation in hospital as well as in general practice

- Individualisation of management

Concept of a diagnostic test for irritable bowel syndrome

Little discussion of cognitive behavioural therapy and other 'talk therapies' 


\section{Appendix 4. Key themes on diagnosis and management from coeliac disease guidelines.}

Early diagnosis including testing at-risk groups with correct serological test

- Structured follow-up/review with practice database/audit, including symptoms, body mass index, relevant bloods (iron, folate)

- Bone mineral density followed by treatment as appropriate (calcium, vitamin $\mathrm{D}$, bisphosphonates)

- Access to/review by dietician

- Appropriate prescription of gluten-free products

- Patient information and Coeliac UK, CORE, Patient UK

Standardisation of serological tests (tissue transglutaminase antibodies)

\section{Appendix 5. Key themes on diagnosis and management} from GORD guidelines.

- Clinical diagnosis on basis of typical symptoms without endoscopy - or if endoscopy negative

- Use Reflux Disease Questionnaire or proton pump inhibitor test to assist diagnosis

- Acid suppression to control symptoms (step-down approach) and ondemand therapy

- Use of GORD Impact Scale to measure response to treatment

- Review co-prescriptions (reflux-inducing drugs)

Patient information: CORE, Patient UK

Establish/evaluate presence of associated non-reflux symptoms

Dietary and other lifestyle (weight, smoking, alcohol) advice and precipitants

\section{Appendix 6. Key themes on diagnosis and management} from irritable bowel syndrome guidelines.

- Early accurate diagnosis using Rome criteria and minimal investigation (full blood count, erythrocyte sedimentation rate, thyroid function tests, tissue transglutaminase antibodies)

- Clear explanation and description of prognosis

- 'Positive diagnosis'

- Recognition of constipation-predominant and diarrhoea-predominant irritable bowel syndrome, with implications for choice of treatment

- Patient information: Gut Trust, CORE, Patient UK

- Psychological assessment (psychopathology associated with poor response to treatment)

Dietary advice: fibre, provocants, fluid, fizzy drinks, fruit (probiotics)

- Symptom-guided treatment, but consider selective serotonin reuptake inhibitor or tricyclic antidepressants at low dose, and talk therapies; for example, cognitive behavioural therapy/hypnotherapy 